\title{
EDITORIALS
}

\section{Provision of health information for all}

\author{
A major organisation should support global efforts
}

\author{
Richard Smith director ${ }^{1}$, Tracey Pérez Koehlmoos programme head ${ }^{2}$ \\ ${ }^{1}$ UnitedHealth Chronic Disease Initiative, London SW4 OLD, UK; ${ }^{2}$ Health and Family Planning Systems Programme, ICDDR,B, Dhaka, Bangladesh
}

High quality information is essential for good health, yet many individuals, practitioners, and health organisations_-particularly in low and middle income countries-lack access to information. This problem has been highlighted many times, ${ }^{1-4}$ and Health Information for All 2015 (HIFA2015) was founded in 2006 with the aim that "by 2015 every person worldwide will have access to an informed healthcare provider-lack of relevant, reliable healthcare information will no longer be a major contributor to avoidable death and suffering" (www.hifa2015. org/). It is unlikely that this ambitious goal will be achieved.

In HIFA2015's definition, the term "healthcare providers" includes mothers and family caregivers, in recognition that their basic knowledge and decisions are crucial to survival. In many countries in Africa more than $80 \%$ of children die before they even reach a health facility. The term "healthcare information" refers to health knowledge for prevention and treatment of disease rather than routine statistical data.

HIFA2015 now has 5000 members from 2000 organisations in 158 countries, and it has four global forums-HIFA2015, CHILD2015, HIFA-Portuguese, and HIFA-EVIPNet. Most of those who contribute to the forums come from low and middle income countries. The organisation has a three pronged strategy of communication (bringing together a critical mass of agents for change), understanding (of information needs and how to meet them), and advocacy (persuading governments, funding agencies, and others to invest in cost effective health information services). There has been progress in access to electronic journals, thanks to initiatives such as the HINARI Access to Research in Health programme and the open access movement, but little progress in meeting the information needs of frontline healthcare providers and ordinary citizens in low resource settings.

The work of HIFA2015 has been achieved with an income of less than $£ 30000$ ( $€ 33300$; $\$ 48000$ ) a year, $£ 10000$ of which comes from the BMA. More than 100 health and development organisations worldwide have committed in principle to the HIFA2015 goal, but no major funding agency has done so. Some $\$ 160$ bn is spent annually on health research, and it seems odd that no organisation will fund improving access to that information. The Rockefeller Foundation is funding an evaluation of HIFA2015 that ironically costs more than the programme being evaluated.

One of the reasons for the lack of commitment may be the lack of clarity of the scale of the problem. The World Health Report 2010 estimated that more than a billion people lack access to healthcare. ${ }^{5}$ There is virtually no access to high quality information in many rural areas of low income countries. The consequences are highlighted in the HIFA2015 report: eight in 10 caregivers in developing countries do not know the two key symptoms of childhood pneumonia, four in 10 mothers in India believe that they should withhold fluids if their baby develops diarrhoea, and three in four doctors caring for sick children in district hospitals in seven low income countries have poor knowledge of the leading causes of death in children. ${ }^{6}$ But these are just anecdotes. A large scale study is needed of the information needs of health providers in low income settings and how well those needs are being met. This could provide the stimulus for global action.

Another reason may be that improving information supply requires more than simply shifting textbooks to Africa or providing internet access. There is a need to create a culture of valuing, generating, and using information-what has been called the knowledge cycle. ${ }^{4}$

Not only is the problem complex it is also not sexy. Funders are much keener to put money into drugs, vaccines, and bed nets than they are into something as nebulous as information access. Yet information and the capacity to act on that information is the first building block of an effective health system, as outlined in WHO's framework for action on health systems strengthening. ${ }^{7}$

Major funders may hope that technology or the private sector will solve the problem. However, internet access is slow and expensive in poorer countries, and it is available to only $11 \%$ of people in Africa, $0.5 \%$ in Ethiopia, and $0.9 \%$ in Bangladesh. ${ }^{8}$ Mobile phones are becoming more available in low income countries, but much more is needed to improve access to information. And although the private sector is the main supplier of information in the rich world, public-private partnerships may well be needed to improve information access in poorer 
countries, in the same way that the Global Alliance for Vaccines and Immunisation was needed to improve access to vaccines.

Fiona Godlee and others called in the Lancet in 2004 for WHO "to take the lead in championing the goal of 'Universal access to essential healthcare information by 2015 ", and for "an international collaborative group along the lines of the Global Fund." Since then WHO has provided moral and technical support for HIFA2015, but it has not led a major initiative and there is no global fund. One of the strengths of HIFA2015 is that it is an international collaborative group that includes the full range of stakeholders, from senior WHO executives in Geneva to rural health workers in Bangladesh, with a predominance of people from low and middle income countries. What it lacks are funds. Rather than start another organisation the best way forward would be for a major funder to recognise that improved health information is fundamental to global health improvement and development and offer substantial support, not just funds, to HIFA2015.

Competing interests: All authors have completed the ICMJE uniform disclosure form at www.icmje.org/coi_disclosure.pdf (available on request from the corresponding author) and declare: no support from any organisation for the submitted work; no financial relationships with any organisations that might have an interest in the submitted work in the previous three years; RS has been involved since the early 1990s in initiatives that improve the availability of health information in developing countries, including HINARI, Public Library of Science, Health Information Forum, and HIFA2015; he attended and spoke at the recent HIFA2015 conference; UnitedHealth Chronic Disease Initiative has applied to be a supporting organisation of HIFA2015; no other relationships or activities that could appear to have influenced the submitted work.

Provenance and peer review: Commissioned; not externally peer reviewed.

1 Kale R. Health information for the developing world. BMJ 1994;309:939-42.

2 Pakenham-Walsh N, Priestly C, Smith R. Meeting the information needs of health workers in developing countries. BMJ 1997:314:90.

3 http://www.ncbi.nlm.nih.gov/sites/entrez?db=pubmed\&cmd=Search\&term=BMJ[Jour] +AND+314[Volume]+AND+90[page]Horton R. North and South: bridging the information gap. Lancet 2000;355:2231-6.

4 Godlee F, Pakenham-Walsh N, Ncayiyan D, Cohen B, Packer A. Can we achieve health information for all by 2015? Lancet 2004;364:295-300.

5 WHO. World health report. Health system financing-the path to universal coverage. 2010. www.who.int/whr/2010/en/index.html.

6 HIFA2015. Annual review 2010. HIFA2015, 2011.

7 WHO. Everybody's business: strengthening health systems to improve health outcomes. WHO's framework for action. 2007. www.who.int/healthsystems/strategy/everybodys business.pdf.

8 Internet World Stats. Internet users in the world. www.internetworldstats.com/stats.htm.

Cite this as: $B M J 2011 ; 342: \mathrm{d} 4151$ 